\title{
RESPONSE OF SOME RICE CULTIVARS TO NITROGEN SOURCE AND TIME OF APPLICATION UNDER SALINE SOIL CONDITIONS
}

\author{
Abd El-Hafez. A. G*; B. A. Zayed ${ }^{\star *}$; I. S. El-Degwy ${ }^{\star}$; A. M. \\ Zayed \\ *Agronomy Department, Fac. of Agric., Kafrelsheikh Univ., Egypt \\ ** Rice Research Section, Field Crop Research Inst, ARC, Giza, Egypt
}

\begin{abstract}
Two filed experiments were conducted during 2013 and 2014 seasons at the Research Farm of El-Sirw Agricultural Research Station Damietta Governorate, Egypt. The experiments were performed to study the response of three rice genotypes namely, Giza178, Giza179, and Egyptian hybrid one (EHR1) to three nitrogen sources viz ammonium sulfate, urea and calcium nitrate and timing of application,[(T1) three equal doses at basal(B), at panicle initiation stage(PI) and at booting stage(BT), (T2) three equal doses at early tillering stage $(\mathrm{T})$, at mid tillering stage(MT) and at BT,(T3) three doses $1 / 2$ at $(\mathrm{T}), 1 / 4$ at (PI) and $1 / 4$ at ( BT) and (T4)four equal doses $1 / 4$ at T, $1 / 4$ at MT, $1 / 4$ at $\mathrm{PI}$ and $1 / 4$ at BT under saline soil conditions]. The experimental soil was clay with salinity levels of 7.5 and $7.3 \mathrm{dSm}^{-1}$ in 2013 and 2014 seasons, respectively. The experiment was performed in split split plot design with four replications. The cultivars were distributed in the main plots, while, the sub plots were allocated to the nitrogen sources. However, the sub sub plots were devoted to times of nitrogen application treatments. The main obtained results could be summarized as follows; the studied rice cultivars showed a significant and marked variation regarding cultivars growth, yield attributes and rice grain yield. Egyptian hybrid rice (EHR1) apparently surpassed the other tested pure line cultivars in growth, yield and yield components in both seasons. The nitrogen source had pronounced effect on rice growth, yield attributes and grain yield in both seasons. The nitrogen in the form of ammonium sulfate showed higher grain yield than the other two nitrogen sources. The time of nitrogen application showed significant growth, yield attributes and grain yield in both seasons. Nitrogen applications into three or four doses (T2, T3 and T4) without basal application (T1) were favorable under saline soil conditions. The interaction effect confirmed the superiority of EHR1, ammonium sulfate and nitrogen application including dose at late growth stage to fetch high reasonable rice grain yield under the same experimental conditions.
\end{abstract}

\section{INTRODUCTION}

Rice (Oryza sativa L.) is the most important crop after wheat. It is a staple food for nearly one half of the world population since most of them live in developing countries. Moreover, it is a very important cereal crop in Egypt for consumption and exportation, in which an 
important source for hard currency. The total rice cultivated area is about 1.54 million fed which produce about 6.08 million tons of paddy rice (RRTC, 2014). Rice is mainly cultivated in the northern Nile-Delta of Egypt, where, salt affected soils are prevailing. Furthermore, poor quality water is used as irrigation water in the target domain area (30$35 \%$ of rice cultivated area) of salt affected soils. The area of saline soil is expected to increase in Egypt as a result of fresh water shortage and climate change. Rice under saline soil needs especial management rather than normal soil. Nitrogen is usually the most yield-limiting nutrient in lowland rice production. Intensive agricultural production systems have increased the use of nitrogen $(\mathrm{N})$ fertilizer. Urea and ammonium sulfate are the two main sources of inorganic $\mathrm{N}$ fertilizer for lowland rice. Nitrogen content is an important criterion; however, other factors should also, be taken into consideration when choosing a fertilizer carrier. These factors include content of nutrients, chemical reactions in soil and nutrient availability to plants in soil. Recovery of $\mathrm{N}$ in crop plants is usually less than $50 \%$ worldwide (Fageria and Baligar, 2005). Worldwide, $\mathrm{N}$ recovery efficiency for cereal production (rice, wheat, sorghum, millet, barley, corn, oat, and rye is approximately $33 \%$. Jan et al., (2010) and Fageria et al. (2011) found that maximum grain yield was obtained at $168 \mathrm{mg} \mathrm{N} \mathrm{kg}^{-1}$ soil in form of ammonium sulfate and at $152 \mathrm{mg} \mathrm{N} \mathrm{kg}^{-1}$ soil as urea. Maximum grain yield at average $\mathrm{N}$ rate $\left(160 \mathrm{mg} \mathrm{kg}^{-1}\right)$ was $22 \%$ higher with the application of ammonium sulfate compared to urea. Rice yield components, $\mathrm{N}$ uptake and use efficiency were significantly a positively influenced with the increase of applied ammonium sulfate. Assefa et al. (2009), Chien et al (2011) and Zayed et al (2012). under saline soil conditions, found that applied ammonium sulfate as nitrogen source significantly surpassed urea application regarding rice growth, LAI, dry matter, chlorophyll content, number of panicles, number of filled grains/panicle, thousand grain weight, panicle weight, grain and straw yields as well as harvest index. Furthermore, applying ammonium sulfate significantly reduced spikelet sterility resulted in heavy panicle and grains as well as higher number of filled grains. Hembram et al. (2001), Balasubramanian (2002), Tao et al. (2002), Edwin et al. (2004) and Zayed et al. (2007). reported that nitrogen application (at basal + active tillering stag + panicle initiation + at panicle emergence) significantly raised dry matter, leaf area index, leaf N\% percentage, chlorophyll content, flag leaf characteristics at heading and markedly enhanced grain yield and yield attribute traits through improving grain filling process. Leaf area index, leaf N\% percentage, growth rate at maturity, dry matter, flag leaf area and its weight and sink capacity were significantly correlated with grain yield of hybrid rice (Yang et al. 1999). Stated that nitrogen application in 
four splits (as basal, topdressing at mid tillering, panicle initiation and booting stags) significantly increased all yield attributes, as well as grain yield of hybrid rice. Therefore, the present study aimed to see the performance of some rice cultivars to different nitrogen sources and time of application under saline soil conditions. to produce high grain yield.

\section{MATERIALS AND METHODS}

Two filed experiments were conducted during of 2013 and 2014 seasons at the Research Farm of El-Sirw Agricultural Research Station, Damietta Governorate, Egypt. The experiments were performed to study response of three rice cultivars to three nitrogen sources and four times of nitrogen application under saline soil conditions. The experiment was performed in split split plot design with four replications. The three rice cultivars, Giza178, Giza179, and Egyptian hybrid one (EHR1) were distributed in the main plots. The three nitrogen sources, ammonium sulfate, urea and calcium nitrate were allocated in the sub-plots, while, the sub-sub plots were devoted to four times of nitrogen application, [(T1) three equal doses at basal(B), at panicle initiation stage (PI) and at booting stage(BT), (T2) three equal doses at early tillering stage $(\mathrm{T})$, at mid tillering stage(MT) and at BT, (T3) three doses $1 / 2$ at (T), $1 / 4$ at (PI) and $1 / 4$ at(BT) and (T4) four equal doses $1 / 4$ at T, $1 / 4$ at MT, $1 / 4$ at $\mathrm{PI}$ and $1 / 4$ at $\mathrm{BT}$ under saline soil conditions]. Representative soils were taken four the experimental sites and analyzed and chemical soil prosperities are listed in Table (1).

Table (1): Chemical soil prosperities of the experimental sites during 2013 and 2014 seasons

\begin{tabular}{|c|c|c|c|c|c|c|c|c|c|}
\hline \multirow[t]{2}{*}{ season } & \multirow[t]{2}{*}{$\mathrm{pH}$} & \multirow{2}{*}{$\begin{array}{c}E C \\
{ }^{1-} \mathrm{dS} \mathrm{m}\end{array}$} & \multicolumn{4}{|c|}{ Cation meq L } & \multicolumn{3}{|c|}{ Anion meq L } \\
\hline & & & $\mathrm{Ca}^{++}$ & $\mathrm{Mg}^{++}$ & $\mathrm{Na}{ }^{+}$ & $\mathrm{K}$ & So4 & $\mathrm{Cl}^{-}$ & $\mathrm{HCO} 3$ \\
\hline 2013 & 8.2 & 7.5 & 18.8 & 16.8 & 40.0 & 0.30 & 32.0 & 40 & 11 \\
\hline 2014 & 8.1 & 7.3 & 19.9 & 16.0 & 39.0 & 0.31 & 30.0 & 43 & 12 \\
\hline \multicolumn{10}{|c|}{ Available nutrients $\mathrm{mg}$} \\
\hline & $\mathrm{N}$ & $\mathrm{P}$ & \multicolumn{2}{|c|}{$\mathrm{K}$} & $\mathrm{Zn}$ & $S$ & $\mathrm{Fe}$ & $\mathrm{Cu}$ & $\mathrm{Mn}$ \\
\hline 2013 & 28.0 & 32.0 & \multicolumn{2}{|c|}{30.0} & 1.08 & 3.0 & 6.0 & 6.2 & 4.1 \\
\hline 2014 & 29.0 & 35.0 & \multicolumn{2}{|c|}{35.0} & 1.01 & 4.0 & 7.0 & 6.0 & 3.8 \\
\hline
\end{tabular}

The nursery was well fertilized with calcium super phosphate $\left(15.5 \% \mathrm{P}_{2} \mathrm{O}_{5}\right)$ at the rate of $71.5 \mathrm{~kg} / \mathrm{ha}^{-1}$ on the dry soil before plaguing. After pudding nitrogen in form of urea $(46 \% \mathrm{~N})$ at the rate of $164.20 \mathrm{~kg} \mathrm{~N} / \mathrm{ha}^{-1}$ and zinc sulfate at the rate of $23.8 \mathrm{~kg}^{-} \mathrm{ha}^{-1}$ were added. Rice seeds at the rate of $142.8 \mathrm{~kg} / \mathrm{ha}^{-1}$ to inbred rice cultivars while, the hybrid rice seeds at the rate of $24 \mathrm{~kg} / \mathrm{ha}^{-1}$ were soaked, in running water, and incubated for 36 hours each to enhance germination pre-germinated seeds were manually broadcasted. Weeds 
were chemically controlled using Saturn (50\%) at the rate of 4.75 liters / ha ${ }^{-1}$ dissolved in 200 liters of water which sprayed using Knapsack sprayer seven days after sowing. Cultivars were sown in separate nursery beds on April, 25th, in the two seasons of study.

The previous crop was Egyptian clover (Trifolium alexandrinum, $\mathrm{L}$ ) in the two seasons of study. The permanent filed soil was well prepared. Calcium super phosphate $\left(15.5 \% \quad \mathrm{P}_{2} \mathrm{O}_{5}\right)$ and potassium sulfate $\left(48 \% \mathrm{~K}_{2} \mathrm{O}\right)$ were applied in the dry soil before flooding. Thirty days old seedling was transplanted $20 \mathrm{~cm}$ row-to-row, 3-4 seedlings/hill with $20 \mathrm{~cm}$ hill to hill. Each plot included ten rows with five-meter length and $20 \mathrm{~cm}$ apart, (area $10 \mathrm{~m}^{2}$ ). The other usual agricultural practices of growing rice were performed as the recommendation of Ministry of Agricultural and Land Reclamation. At heading stage plant samples (five hills each), were randomly taken from each plot to estimate the following; Leaf area index, dry matter weight $(\mathrm{g}) / \mathrm{m}^{2}$, chlorophyll content and heading date(days). Plant height $(\mathrm{cm})$ was measured from the soil surface up to the tallest panicle tip for each plant. (productive and non-productive tillers) of five hills were estimated and then converted to number of tillers/hill. At harvest, ten main panicles were randomly taken to determine the grain yield attributes; i.e., panicle length $(\mathrm{cm})$, number of panicles / hill, number of filled grains / panicle, number of unfilled grains/panicle, panicle weight (g) and 1000-grain weight (g). The grain and straw yields, of six inner rows for each sub plot, were determined and converted into $t / \mathrm{ha}^{-1}$, based on $14 \%$ moisture content. All the data collected were subjected to analysis of variance according to Gomez and Gomez (1984). Treatment means were compared by Duncan's Multiple Range Test (Duncan, 1955). All statistical analysis were performed using analysis of variance technique by means of "COSTAT" computer soflwar package.

\section{1- Rice cultivars performance:}

\section{RESULTS AND DISCUSSION}

The rice cultivars significantly differed in their growth, yield and grain yield components in both seasons. The Egyptian hybrid rice one (EHR1) variety obviously surpassed the other two rice varieties Tables 2 and 3. It had high hetrosis in dry matter production, leaf area index, chlorophyll content, plant height, number of tillers, number of filled grains, number of unfilled spikelet, panicle length, number of panicles, panicle weight, 1000-grain weight, biological and grain yields in both seasons. The EHR1 and Giza178 did not differ in heading date in both seasons. Giza178 and Giza179 rice cultivars were at apart regarding to dry matter production, chlorophyll content, number of unfilled spikelet and biological yield in both seasons, as well as leaf area index 
and grain yield in 2013 season and number of tillers, panicle weight in 2014 season. The cultivars (Giza178 and Giza179) recorded lower values in dry matter production, chlorophyll content, number of unfilled spikelet and biological yield in both seasons compared to the hybrid (EHR1). Giza179 gave the lowest values of leaf area index, number of panicles and grain yield in 2014 season. However, Giza 179 recorded the lowest number of days to heading, plant height, number of filled grains and panicle length in both seasons. EHR1 hybrid showed its superiority in the above-mentioned traits while, Giza178 and Giza179 cultivars were in the last rank regarding the above-mentioned traits in both seasons. The superiority of the hybrid rice cultivar was mainly due to the high heterosis than that is not found in the cultivars Giza178 and Giza179. Similar findings had been reported by El-Refaee et al (2007), Zayed et al (2007) and Zayed et al (2010).

\section{2- Effect of nitrogen source}

Data in Tables 2 and 3 showed that nitrogen sources had a significant effect on all the studied traits except for heading date in both seasons. Ammonium sulfate and urea were at the same level of significance in leaf area index, chlorophyll content, number of unfilled grains, panicle length, 1000-grain weight and grain yield in both seasons. Plant height, number of tillers, number of filled grains/panicle, panicle weight and biological yield in 2013 season had the same previous pattern.

The nitrogen in the form of ammonium sulfate obviously was superior in dry matter production, number of panicles in both seasons and plant height, number of tillers, number of filled grains, panicle weight and biological yield in 2014 season compared to other two nitrogen sources. Meanwhile, the lowest values of all above-mentioned traits were recorded by calcium nitrate in 2013 and 2014 seasons. Ammonium sulfate as source for both of nitrogen and sulfur assimilated might have played a vital role in growth and development of rice plants because of their active role in plant metabolic processes. Also, application of ammonium sulfate might improve soil physical and chemical proprieties, under current saline soil with high $\mathrm{pH}$ which, in turn, resulted in improving nutrients availability, low $\mathrm{pH}$ and bulk density leading to increase rice salt tolerance, improve rice growth, proper yield components and subsequently high yield. As seen ammonium sulfate showed its superiority in most of studied traits in both seasons that can be attributed to the readily soluble nature of the former. Similar findings were reported by. Assefa et al. (2009) Chien et al (2011) and Zayed et al (2012). 


\section{3-Time of nitrogen application:}

Time of nitrogen application treatments were significantly affected rice growth, dry matter, LAI, yield attributes as well as grain yield in both seasons. It is brought that the treatment including basal application did not apparently improve rice growth and grain yield as well as yield attributes. The model including basal application gave the lowest value of above-mentioned traits. Splitting nitrogen into three (T3) and four doses (T4) exerted high dry matter in 2013 and 2014 seasons respectively.

Table (2): Some growth characteristics of rice cultivars as affected by nitrogen sources and time of application in 2013 and 2014 seasons

\begin{tabular}{|c|c|c|c|c|c|c|c|c|c|c|c|c|}
\hline \multirow{2}{*}{ Treatment } & \multicolumn{2}{|c|}{ Dry matter $(\mathbf{g}) \mathrm{m}^{-2}$} & \multicolumn{2}{|c|}{ Leaf area index } & \multicolumn{2}{|c|}{ Chlorophyll content } & \multicolumn{2}{|c|}{ Days to heading } & \multicolumn{2}{|c|}{ Plant height $(\mathrm{cm})$} & \multicolumn{2}{|c|}{$\begin{array}{c}\text { Number of tillers } \\
\text { hill }^{-1}\end{array}$} \\
\hline & 2013 & 2014 & 2013 & 2014 & 2013 & 2014 & 2013 & 2014 & 2013 & 2014 & 2013 & 2014 \\
\hline $\begin{array}{r}\text { Cultivar (C): } \\
\text { Giza } 178 \\
\text { Giza } 179 \\
\text { EHR1 }\end{array}$ & $\begin{array}{c}901.8 \mathrm{~b} \\
932.5 \mathrm{~b} \\
1071.1 \\
\mathrm{a}\end{array}$ & $\begin{array}{c}1023.7 \\
b \\
1085.3 \\
b \\
1177.8 \\
a \\
\end{array}$ & $\begin{array}{l}3.56 \mathrm{~b} \\
3.51 \mathrm{c} \\
4.44 \mathrm{a}\end{array}$ & $\begin{array}{l}3.75 \mathrm{~b} \\
3.51 \mathrm{~b} \\
4.41 \mathrm{a}\end{array}$ & $\begin{array}{l}40.65 \mathrm{~b} \\
40.45 \mathrm{~b} \\
43.51 \mathrm{a}\end{array}$ & $\begin{array}{l}42.78 \mathrm{~b} \\
42.33 \mathrm{~b} \\
43.95 \mathrm{a}\end{array}$ & $\begin{array}{l}99.31 \mathrm{a} \\
90.39 \mathrm{~b} \\
98.66 \mathrm{a}\end{array}$ & $\begin{array}{l}104.01 a \\
92.35 b \\
102.97 a\end{array}$ & $\begin{array}{l}86.27 b \\
80.27 \mathrm{c} \\
95.49 \mathrm{a}\end{array}$ & $\begin{array}{l}90.32 \mathrm{~b} \\
82.76 \mathrm{c} \\
98.14 \mathrm{a}\end{array}$ & $\begin{array}{l}16.89 \mathrm{c} \\
18.75 \mathrm{~b} \\
20.24 \mathrm{a}\end{array}$ & $\begin{array}{l}21.65 b \\
22.06 b \\
22.85 a\end{array}$ \\
\hline F. test & $* *$ & $* *$ & $* *$ & ** & $* *$ & $* *$ & ** & $* *$ & $* *$ & $* *$ & $* *$ & $* \star$ \\
\hline $\begin{array}{c}\frac{\mathbf{N} \text { source (S): }}{\text { Ammonium sulfate }} \\
\text { Urea } \\
\text { Calcium nitrate }\end{array}$ & $\begin{array}{c}1054.2 \\
a \\
995.4 \mathrm{~b} \\
855.7 \mathrm{c} \\
\end{array}$ & $\begin{array}{c}1168.4 \\
a \\
1096.1 \\
b \\
1022.4 \\
c \\
\end{array}$ & $\begin{array}{l}3.96 \mathrm{a} \\
4.19 \mathrm{a} \\
3.34 \mathrm{~b} \\
\end{array}$ & $\begin{array}{l}4.05 a \\
4.27 a \\
3.34 b \\
\end{array}$ & $\begin{array}{l}42.47 a \\
41.82 a \\
40.31 b \\
\end{array}$ & $\begin{array}{l}43.60 \mathrm{a} \\
43.19 \mathrm{a} \\
42.27 \mathrm{~b} \\
\end{array}$ & $\begin{array}{l}95.77 \\
96.41 \\
96.18 \\
\end{array}$ & $\begin{array}{l}99.78 \\
99.82 \\
99.73 \\
\end{array}$ & $\begin{array}{l}89.49 a \\
90.73 a \\
82.17 b \\
\end{array}$ & $\begin{array}{l}93.22 \mathrm{a} \\
91.73 \mathrm{~b} \\
86.27 \mathrm{c} \\
\end{array}$ & $\begin{array}{l}19.36 \mathrm{a} \\
19.10 \mathrm{a} \\
17.41 \mathrm{~b} \\
\end{array}$ & $\begin{array}{l}22.83 \mathrm{a} \\
22.15 \mathrm{~b} \\
21.58 \mathrm{c} \\
\end{array}$ \\
\hline F. test & $* *$ & ** & $* *$ & ** & $* *$ & ${ }^{* *}$ & NS & NS & $* *$ & ${ }^{* *}$ & $* *$ & $* *$ \\
\hline $\begin{array}{c}\text { Time of N application }(\mathrm{T}): \\
1 / 3(\mathrm{~B}+\mathrm{PI}+\mathrm{BT}) \\
1 / 3(\mathrm{~T}+\mathrm{MT}+\mathrm{BT}) \\
1 / 2 \mathrm{~T}+1 / 4 \mathrm{PI}+1 / 4 \mathrm{BT} \\
1 / 4(\mathrm{~T}+\mathrm{MT}+\mathrm{PI}+\mathrm{BT})\end{array}$ & $\begin{array}{l}888.1 \mathrm{c} \\
979.9 \mathrm{~b} \\
1032.1 \\
\mathrm{a} \\
973.6 \mathrm{~b}\end{array}$ & $\begin{array}{c}1014.4 \\
b \\
1120.9 \\
a \\
1121.6 \\
a \\
1125.7 \\
a \\
\end{array}$ & $\begin{array}{l}3.50 \mathrm{c} \\
3.79 \mathrm{~b} \\
3.92 \mathrm{a} \\
4.12 \mathrm{a}\end{array}$ & $\begin{array}{l}3.55 \mathrm{~b} \\
3.91 \mathrm{ab} \\
4.02 \mathrm{a} \\
4.12 \mathrm{a}\end{array}$ & $\begin{array}{l}41.31 \\
41.57 \\
41.45 \\
41.81\end{array}$ & $\begin{array}{l}43.00 \\
43.15 \\
43.16 \\
42.77\end{array}$ & $\begin{array}{l}95.80 \\
96.11 \\
96.27 \\
96.30\end{array}$ & $\begin{array}{c}99.34 \\
99.75 \\
100.15 \\
99.87\end{array}$ & $\begin{array}{l}85.61 \mathrm{~b} \\
86.44 \mathrm{~b} \\
88.81 \mathrm{a} \\
88.51 \mathrm{a}\end{array}$ & $\begin{array}{l}89.64 \\
90.12 \\
91.21 \\
90.66\end{array}$ & $\begin{array}{l}17.90 \mathrm{~b} \\
18.77 \mathrm{a} \\
19.20 \mathrm{a} \\
18.63 \mathrm{a}\end{array}$ & $\begin{array}{l}21.90 \mathrm{~b} \\
22.66 \mathrm{a} \\
22.38 \mathrm{ab} \\
22.81 \mathrm{a}\end{array}$ \\
\hline F. test & ** & ** & *夫 & ** & NS & NS & NS & NS & ** & NS & ** & ** \\
\hline $\begin{array}{l}\text { Interaction } \\
C \times S \\
C \times T \\
T \times S \\
C \times T \times S\end{array}$ & $\begin{array}{l}* * \\
* * \\
* * \\
\text { NS }\end{array}$ & $\begin{array}{l}\text { NS } \\
\text { NS } \\
\text { NS } \\
\text { NS }\end{array}$ & $\begin{array}{l}\text { NS } \\
\text { NS } \\
\text { NS } \\
\text { NS }\end{array}$ & $\begin{array}{l}\text { NS } \\
\text { NS } \\
\text { NS } \\
\text { NS }\end{array}$ & $\begin{array}{l}\text { NS } \\
* * \\
\text { NS } \\
\text { NS }\end{array}$ & $\begin{array}{l}\text { NS } \\
\text { NS } \\
\text { NS } \\
\text { NS }\end{array}$ & $\begin{array}{l}\text { NS } \\
\text { NS } \\
\text { NS } \\
\text { NS }\end{array}$ & $\begin{array}{l}\text { NS } \\
\text { NS } \\
\text { NS } \\
\text { NS }\end{array}$ & $\begin{array}{l}* * \\
* * \\
N S \\
\text { NS }\end{array}$ & $\begin{array}{c}* \star \\
* \star \\
* \star \\
\text { NS }\end{array}$ & $\begin{array}{l}\text { NS } \\
\text { NS } \\
\star * \\
\text { NS }\end{array}$ & $\begin{array}{l}\text { NS } \\
\text { NS } \\
* * \\
\text { NS }\end{array}$ \\
\hline
\end{tabular}

NS $=$ not significant and ${ }^{* *}$ significant at $1 \%$ levels.

$\mathrm{T}=$ beginning of tillering stage, $\mathrm{MT}=$ mid tillering stage, $\mathrm{PI}=$ panicle initiation stage and $\mathrm{BT}=$ booting stage 
Table (3): Grain yield and some of its attributes of rice cultivars as affected by nitrogen sources and times of application in 2013 and 2014 seasons

\begin{tabular}{|c|c|c|c|c|c|c|c|c|c|c|c|c|c|c|c|c|}
\hline \multirow{2}{*}{ Treatment } & \multicolumn{2}{|c|}{$\begin{array}{c}\text { Number of } \\
\text { panicles/hill }\end{array}$} & \multicolumn{2}{|c|}{$\begin{array}{l}\text { Panicle weight } \\
\text { (g) }\end{array}$} & \multicolumn{2}{|c|}{$\begin{array}{l}\text { Panicle length } \\
(\mathrm{cm})\end{array}$} & \multicolumn{2}{|c|}{$\begin{array}{l}\text { Number of filled } \\
\text { grains/panicle }\end{array}$} & \multicolumn{2}{|c|}{$\begin{array}{c}\text { Number of } \\
\text { unfilled } \\
\text { grains/panicle }\end{array}$} & \multicolumn{2}{|c|}{$\begin{array}{l}\text { 1000-grain } \\
\text { weight }(\mathrm{g})\end{array}$} & \multicolumn{2}{|c|}{$\begin{array}{l}\text { Biological yield } \\
\text { (t/ha) }\end{array}$} & \multicolumn{2}{|c|}{ Grain yield ( $t / h a)$} \\
\hline & 2013 & 2014 & 2013 & 2014 & 2013 & 2014 & 2013 & 2014 & 2013 & 2014 & 2013 & 2014 & 2013 & 2014 & 2013 & 2014 \\
\hline $\begin{array}{r}\text { Cultivar (C): } \\
\text { Giza } 178 \\
\text { Giza } 179 \\
\text { EHR1 }\end{array}$ & $\begin{array}{l}14.40 \mathrm{c} \\
16.00 \mathrm{~b} \\
17.53 \mathrm{a}\end{array}$ & $\begin{array}{l}17.72 \mathrm{~b} \\
16.39 \mathrm{c} \\
18.84 \mathrm{a}\end{array}$ & $\begin{array}{l}2.52 \mathrm{c} \\
2.83 \mathrm{~b} \\
3.16 \mathrm{a}\end{array}$ & $\begin{array}{l}3.07 \mathrm{~b} \\
3.11 \mathrm{~b} \\
3.94 \mathrm{a}\end{array}$ & $\begin{array}{l}20.18 \mathrm{~b} \\
17.85 \mathrm{c} \\
21.70 \mathrm{a}\end{array}$ & $\begin{array}{l}20.91 \mathrm{~b} \\
18.45 \mathrm{c} \\
21.94 \mathrm{a}\end{array}$ & $\begin{array}{c}108.6 \mathrm{~b} \\
92.5 \mathrm{c} \\
122.0 \mathrm{a}\end{array}$ & $\begin{array}{l}123.9 \mathrm{~b} \\
104.1 \mathrm{c} \\
127.1 \mathrm{a}\end{array}$ & $\begin{array}{l}18.3 \mathrm{~b} \\
17.9 \mathrm{~b} \\
26.5 \mathrm{a}\end{array}$ & $\begin{array}{l}16.8 \mathrm{~b} \\
16.4 \mathrm{~b} \\
19.3 \mathrm{a}\end{array}$ & $\begin{array}{l}18.50 \mathrm{c} \\
22.69 \mathrm{~b} \\
23.04 \mathrm{a}\end{array}$ & $\begin{array}{l}20.65 \mathrm{c} \\
23.82 \mathrm{~b} \\
24.54 \mathrm{a}\end{array}$ & $\begin{array}{l}13.04 \mathrm{~b} \\
13.99 \mathrm{~b} \\
16.15 \mathrm{a}\end{array}$ & $\begin{array}{l}14.18 \mathrm{~b} \\
14.78 \mathrm{~b} \\
17.41 \mathrm{a}\end{array}$ & $\begin{array}{l}6.38 \mathrm{~b} \\
6.15 \mathrm{~b} \\
7.18 \mathrm{a}\end{array}$ & $\begin{array}{l}6.55 \mathrm{~b} \\
6.29 \mathrm{c} \\
7.40 \mathrm{a}\end{array}$ \\
\hline F. test & $* \star$ & $* *$ & $* *$ & $\star * *$ & $* \star *$ & $* *$ & $\star \star \star *$ & $* \star$ & $* *$ & $* *$ & $* *$ & 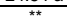 & $\star \star *$ & $* \star$ & $\star *$ & $* *$ \\
\hline $\begin{array}{c}\mathbf{N} \text { source }(\mathbf{S}): \\
\text { Ammonium sulfate } \\
\text { Urea } \\
\text { Calcium nitrate }\end{array}$ & $\begin{array}{l}17.27 \mathrm{a} \\
16.42 \mathrm{~b} \\
14.24 \mathrm{c} \\
\end{array}$ & $\begin{array}{l}19.31 \mathrm{a} \\
18.32 \mathrm{~b} \\
15.33 \mathrm{c}\end{array}$ & $\begin{array}{l}2.99 \mathrm{a} \\
2.94 \mathrm{a} \\
2.58 \mathrm{~b} \\
\end{array}$ & $\begin{array}{l}3.71 \mathrm{a} \\
3.50 \mathrm{~b} \\
2.92 \mathrm{c} \\
\end{array}$ & $\begin{array}{l}20.30 a \\
20.13 a \\
19.30 b\end{array}$ & $\begin{array}{l}20.94 \mathrm{a} \\
20.62 \mathrm{a} \\
19.73 \mathrm{~b} \\
\end{array}$ & $\begin{array}{c}113.8 \mathrm{a} \\
109.4 \mathrm{a} \\
99.9 \mathrm{~b}\end{array}$ & $\begin{array}{l}124.0 \mathrm{a} \\
121.1 \mathrm{~b} \\
110.4 \mathrm{c} \\
\end{array}$ & $\begin{array}{l}17.1 \mathrm{~b} \\
17.9 \mathrm{~b} \\
27.6 \mathrm{a}\end{array}$ & $\begin{array}{l}15.0 \mathrm{~b} \\
15.0 \mathrm{~b} \\
22.6 \mathrm{a}\end{array}$ & $\begin{array}{l}21.98 a \\
21.70 a \\
20.55 b \\
\end{array}$ & $\begin{array}{l}23.43 \mathrm{a} \\
23.06 \mathrm{a} \\
22.51 \mathrm{~b}\end{array}$ & $\begin{array}{l}15.11 \mathrm{a} \\
14.63 \mathrm{a} \\
13.44 \mathrm{~b} \\
\end{array}$ & $\begin{array}{l}16.60 \mathrm{a} \\
15.51 \mathrm{~b} \\
14.27 \mathrm{c} \\
\end{array}$ & $\begin{array}{l}7.05 \mathrm{a} \\
6.99 \mathrm{a} \\
5.68 \mathrm{~b} \\
\end{array}$ & $\begin{array}{l}7.16 \mathrm{a} \\
7.08 \mathrm{a} \\
5.99 \mathrm{~b}\end{array}$ \\
\hline F. test & $* \star$ & ** & $\star *$ & $\star \star \star *$ & ** & $* *$ & $* \star$ & ** & $* \star$ & ** & $\star * *$ & $* *$ & $* *$ & $\star *$ & $\star \star *$ & ** \\
\hline $\begin{array}{c}\text { Time of } \mathrm{N} \text { application }(\mathrm{T}): \\
1 / 3(\mathrm{~B}+\mathrm{PI}+\mathrm{BT}) \\
1 / 3(\mathrm{~T}+\mathrm{MT}+\mathrm{BT}) \\
1 / 2 \mathrm{~T}+1 / 4 \mathrm{PI}+1 / 4 \mathrm{BT} \\
1 / 4(\mathrm{~T}+\mathrm{MT}+\mathrm{PI}+\mathrm{BT})\end{array}$ & $\begin{array}{l}15.34 \mathrm{~b} \\
16.25 \mathrm{a} \\
16.00 \mathrm{ab} \\
16.32 \mathrm{a}\end{array}$ & $\begin{array}{l}17.00 \mathrm{~b} \\
17.94 \mathrm{a} \\
17.73 \mathrm{a} \\
17.94 \mathrm{a}\end{array}$ & $\begin{array}{l}2.68 \mathrm{~b} \\
2.91 \mathrm{a} \\
2.86 \mathrm{a} \\
2.89 \mathrm{a}\end{array}$ & $\begin{array}{l}2.99 \mathrm{c} \\
3.42 \mathrm{~b} \\
3.51 \mathrm{ab} \\
3.60 \mathrm{a} \\
\end{array}$ & $\begin{array}{l}19.88 \\
19.98 \\
20.11 \\
19.66 \\
\end{array}$ & $\begin{array}{l}20.26 \mathrm{~b} \\
20.71 \mathrm{a} \\
20.45 \mathrm{ab} \\
20.30 \mathrm{~b}\end{array}$ & $\begin{array}{l}100.3 \mathrm{~b} \\
110.4 \mathrm{a} \\
108.6 \mathrm{a} \\
111.6 \mathrm{a}\end{array}$ & $\begin{array}{l}113.1 d \\
115.2 \mathrm{c} \\
120.4 \mathrm{~b} \\
124.8 \mathrm{a}\end{array}$ & $\begin{array}{l}27.4 \mathrm{a} \\
20.8 \mathrm{~b} \\
19.4 \mathrm{c} \\
15.9 \mathrm{~d}\end{array}$ & $\begin{array}{l}23.3 \mathrm{a} \\
17.7 \mathrm{~b} \\
16.0 \mathrm{c} \\
13.0 \mathrm{~d}\end{array}$ & $\begin{array}{l}20.88 \mathrm{c} \\
21.27 \mathrm{~b} \\
21.61 \mathrm{a} \\
21.89 \mathrm{a} \\
\end{array}$ & $\begin{array}{l}22.33 \mathrm{~b} \\
23.28 \mathrm{a} \\
23.26 \mathrm{a} \\
23.15 \mathrm{a}\end{array}$ & $\begin{array}{l}12.23 \mathrm{c} \\
15.01 \mathrm{ab} \\
14.57 \mathrm{~b} \\
15.77 \mathrm{a} \\
\end{array}$ & $\begin{array}{l}13.05 \mathrm{c} \\
16.19 \mathrm{~b} \\
15.60 \mathrm{~b} \\
16.99 \mathrm{a} \\
\end{array}$ & $\begin{array}{l}6.31 \mathrm{~b} \\
6.68 \mathrm{a} \\
6.58 \mathrm{ab} \\
6.73 \mathrm{a}\end{array}$ & $\begin{array}{l}6.36 \mathrm{~b} \\
6.84 \mathrm{a} \\
6.83 \mathrm{a} \\
6.94 \mathrm{a}\end{array}$ \\
\hline F. test & 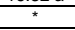 & * & $\star *$ & $\star *$ & NS & * & ** & $\star *$ & $* *$ & $* *$ & $\star * *$ & $\star *$ & $* *$ & $* *$ & $* *$ & ** \\
\hline $\begin{array}{l}\text { Interaction } \\
C \times S \\
C \times T \\
T \times S \\
C \times T \times S\end{array}$ & $\begin{array}{l}\text { NS } \\
\text { NS } \\
\text { NS }\end{array}$ & $\begin{array}{l}* * \\
N S \\
\text { NS }\end{array}$ & $\begin{array}{l}\text { NS } \\
* * \\
\text { NS } \\
\text { NS }\end{array}$ & $\begin{array}{l}* * \\
N S \\
\text { NS }\end{array}$ & $\begin{array}{l}\text { NS } \\
\text { NS } \\
\text { NS } \\
\text { NS }\end{array}$ & $\begin{array}{l}\text { NS } \\
\text { NS } \\
\text { NS } \\
\text { NS }\end{array}$ & $\begin{array}{l}\text { NS } \\
\text { NS } \\
\text { NS }\end{array}$ & $\begin{array}{l}\text { NA } \\
\text { ** } \\
* *\end{array}$ & $\begin{array}{l}\star \star \\
\star * \\
\star * \\
\star \star\end{array}$ & $\begin{array}{c}\text { ** } \\
\text { NS } \\
* * \\
* *\end{array}$ & $\begin{array}{l}* * \\
* * \\
* * \\
* *\end{array}$ & $\begin{array}{l}\text { NS } \\
\text { NS } \\
\text { NS } \\
\text { NS }\end{array}$ & $\begin{array}{l}\text { NS } \\
\text { NS } \\
\text { NS }\end{array}$ & $\begin{array}{l}\text { NS } \\
\text { NS } \\
{ }^{*} \\
\text { NS }\end{array}$ & $\begin{array}{l}\text { ** } \\
\text { NS } \\
\text { NS } \\
\text { NS }\end{array}$ & $\begin{array}{c}\star \star \\
\star \star \\
\star \star \\
\text { NS }\end{array}$ \\
\hline
\end{tabular}

NS $=$ not significant, ${ }^{*}$ and ${ }^{* *}$ significant at 5 and $1 \%$ levels, respectively.

$\mathrm{T}=$ beginning of tillering stage, $\mathrm{MT}=$ mid tillering stage, $\mathrm{PI}=$ panicle initiation stage and $\mathrm{BT}=$ booting stage

While four splits (T4) gave the largest leaf area index without significant differences between each other in chlorophyll content and heading date in both seasons. Plant height in the first season did not significantly response to timing of nitrogen models application. The highest panicle number was produced when rice plants received their nitrogen in the formula of $1 / 2 \mathrm{~T}+1 / 4 \mathrm{PI}+1 / 4$ BT stage without significant differences with equal splits at $\mathrm{T}+\mathrm{MT}+\mathrm{BT}$ and four equal splits at $\mathrm{T}+$ $\mathrm{MT}+\mathrm{PI}+\mathrm{BT}$ gave the best panicle characteristics, filled grains, panicle weight and thousand grain weight reflecting on rice grain biological yields.Applying nitrogen at four equal splits might ensure nitrogen supply with optimum rate at most salt sensitive growth stages such as mid tillering, panicle initiation and mid booting stage that increase rice salinity tolerance and improve rice growth and photosynthesis. Appling small nitrogen dose at late growth stage in the terms of mid booting stage might had increased the concentration of cytokines and rubisco enzyme in root and shoot, respectively resulted in delaying and relieving early aging happening under salt stress. Reliving early aging in rice by adding late nitrogen improve grain filling leading to panicle characteristics improvement and giving high rice grain yield. Similar findings were reported by. Balasubramanian (2002), Tao et al. (2002), Edwin et al. (2004) and Zayed et al (2007). 4-Interaction effect:

The interaction between rice cultivars and nitrogen sources, cultivars and times of nitrogen application and nitrogen sources and time of nitrogen application had significant effect on dry matter only in 2013 season data presented in Table (4). The best combination was 
Egyptian hybrid one and ammonium sulfate nitrogen form since it gave the highest values of dry matter production. For the interaction between cultivars and times of nitrogen application, EHR1 gave the highest mean of dry matter production when plants were received its nitrogen in the following formula $1 / 2 \mathrm{~T}+1 / 4 \mathrm{PI}+1 / 4 \mathrm{BT}$. For the interaction between nitrogen sources and times of nitrogen application it being that ammonium sulfate nitrogen form recorded it is higher dry matter production when it was splited as half at tillering stage plus the rest in two equal dose at panicle initiation + mid of booting stag. The interaction effect data came to confirm the inferiority of basal application of nitrogen under saline soil for both traditional and hybrid rice regarding their physiological traits at heading stag. Furthermore, one third or one fourth of recommended nitrogen at the beginning of heading could be essential for rice growth under saline soil for both hybrid and traditional rice.

The interaction between cultivars and times of nitrogen application had significant effect on chlorophyll content only in 2013 season results presented in Table (4). Egyptian hybrid 1gave the highest values of chlorophyll content under three equal nitrogen splits $1 / 2 T+1 / 4 \mathrm{PI}+1 / 4 \mathrm{BT}$ and $1 / 3 \mathrm{~T}+1 / 3 \mathrm{MT}+1 / 3 \mathrm{BT}$.

The interaction between cultivars and nitrogen sources, cultivars and times of nitrogen application and nitrogen sources had significant effect on plant height in 2013 and 2014 seasons also, time of nitrogen application had significant effect on plant height only in 2014 season data presented in Table (4). The best combination was Egyptian hybrid one and ammonium sulfate nitrogen form since it gave the tallest of plant height. For the interaction between cultivars and times of nitrogen application, EHR1 gave the tallest of plant height when plants were received its nitrogen in the following formula $1 / 4 T+$ $1 / 4 \mathrm{MT}+1 / 4 \mathrm{PI}+1 / 4 \mathrm{BT}$. For the interaction between nitrogen sources and times of nitrogen application it being that ammonium sulfate nitrogen form recorded it is higher plant height when it was splited as half at tillering stage plus the rest in two equal dose at panicle initiation + mid of booting stag.

Data in Table (4) indicated that the interaction between nitrogen sources and time of nitrogen application had significant effect on number of tillers in both seasons. The combination of ammonium sulfate added $1 / 3 T+1 / 3 \mathrm{MT}+1 / 3$ BT gave the highest number of tillers in both seasons.

Data in Table (5) indicated that the interaction between cultivars and nitrogen sources had significant effect on number of panicles only in 2014 season also, interaction between nitrogen sources and times of nitrogen application had significant effect on number of panicles in 2013 and 2014 seasons. The combination of 
Egyptian hybrid one and ammonium sulfate gave the highest values of number of panicles. For the interaction between nitrogen sources and times of nitrogen application the combination of ammonium sulfate under $1 / 3 T+1 / 3 \mathrm{MT}+1 / 3$ BT gave the highest number of tillers in both seasons.

Table (4): Dry matter, chlorophyll content, plant height and number of tillers as affected by the interaction between the studied factors

\begin{tabular}{|c|c|c|c|c|c|c|c|}
\hline \multirow{6}{*}{ 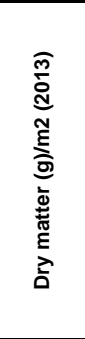 } & \multirow{2}{*}{ N source } & \multicolumn{6}{|c|}{ Rice cultivar } \\
\hline & & \multicolumn{2}{|c|}{ Giza 178} & \multicolumn{2}{|c|}{ Giza 179} & \multicolumn{2}{|c|}{ EHR1 } \\
\hline & $\begin{array}{c}\text { Ammonium sulfate } \\
\text { Urea } \\
\text { Calcium nitrate }\end{array}$ & \multicolumn{2}{|c|}{$\begin{array}{l}968.2 \mathrm{c} \\
903.9 \mathrm{~d} \\
833.4 \mathrm{e}\end{array}$} & \multicolumn{2}{|c|}{$\begin{array}{l}999.3 \mathrm{c} \\
966.3 \mathrm{c} \\
861.8 \mathrm{e}\end{array}$} & \multicolumn{2}{|c|}{$\begin{array}{l}1195.2 \mathrm{a} \\
1115.9 \mathrm{~b} \\
902.1 \mathrm{~d}\end{array}$} \\
\hline & \multirow[b]{2}{*}{ Time of $\mathrm{N}$ application } & \multicolumn{3}{|c|}{ Rice cultivar } & \multicolumn{3}{|c|}{ N source } \\
\hline & & Giza 178 & Giza 179 & EHR1 & A. sulfate & Urea & $\begin{array}{c}\text { Calcium } \\
\text { nitrate }\end{array}$ \\
\hline & $\begin{array}{c}1 / 3(B+P I+B T) \\
1 / 3(T+M T+B T) \\
1 / 2 T+1 / 4 P I+1 / 4 B T \\
1 / 4(T+M T+P I+B T) \\
\end{array}$ & $\begin{array}{l}848.4 \mathrm{f} \\
885.7 \text { ef } \\
988.0 \mathrm{c} \\
885.1 \text { ef }\end{array}$ & $\begin{array}{l}884.0 \mathrm{ef} \\
917.7 \mathrm{de} \\
968.6 \mathrm{~cd} \\
959.6 \mathrm{~cd}\end{array}$ & $\begin{array}{l}931.9 \mathrm{de} \\
1136.3 \mathrm{a} \\
1140.0 \mathrm{a} \\
1076.0 \mathrm{~b}\end{array}$ & $\begin{array}{c}941.5 \mathrm{c} \\
1080.7 \mathrm{ab} \\
1127.9 \mathrm{a} \\
1066.8 \mathrm{~b} \\
\end{array}$ & $\begin{array}{l}866.1 \mathrm{~d} \\
1027.0 \mathrm{~b} \\
1037.5 \mathrm{~b} \\
1050.9 \mathrm{~b}\end{array}$ & $\begin{array}{c}856.7 \mathrm{~d} \\
832.1 \mathrm{de} \\
931.2 \mathrm{c} \\
802.9 \mathrm{c}\end{array}$ \\
\hline \multirow{3}{*}{ 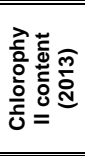 } & \multirow{2}{*}{ Time of $\mathbf{N}$ application } & \multicolumn{6}{|c|}{ Rice cultivar } \\
\hline & & \multicolumn{2}{|c|}{ Giza 178} & \multicolumn{2}{|c|}{ Giza 179} & \multicolumn{2}{|c|}{ EHR1 } \\
\hline & $\begin{array}{c}1 / 3(B+P I+B T) \\
1 / 3(T+M T+B T) \\
1 / 2 T+1 / 4 P I+1 / 4 B T \\
1 / 4(T+M T+P I+B T) \\
\end{array}$ & \multicolumn{2}{|c|}{$\begin{array}{l}39.30 \mathrm{~d} \\
40.26 \mathrm{~cd} \\
41.34 \mathrm{bc} \\
41.69 \mathrm{bc}\end{array}$} & \multicolumn{2}{|c|}{$\begin{array}{l}40.13 \mathrm{~cd} \\
39.97 \mathrm{~cd} \\
40.16 \mathrm{bc} \\
41.54 \mathrm{bc}\end{array}$} & \multicolumn{2}{|c|}{$\begin{array}{c}44.50 \mathrm{a} \\
44.49 \mathrm{a} \\
42.85 \mathrm{ab} \\
42.20 \mathrm{~b}\end{array}$} \\
\hline \multirow{9}{*}{ 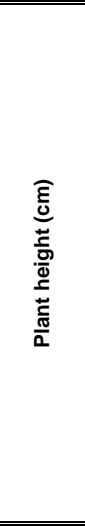 } & \multirow{3}{*}{ N source } & \multicolumn{6}{|c|}{ Rice cultivar } \\
\hline & & \multicolumn{3}{|c|}{2013} & \multicolumn{3}{|c|}{2014} \\
\hline & & Giza 178 & Giza 179 & EHR1 & Giza 178 & Giza 179 & EHR1 \\
\hline & $\begin{array}{c}\text { Ammonium sulfate } \\
\text { Urea } \\
\text { Calcium nitrate } \\
\end{array}$ & $\begin{array}{l}85.68 \mathrm{c} \\
88.43 \mathrm{~b} \\
84.68 \mathrm{~cd} \\
\end{array}$ & $\begin{array}{l}82.97 \mathrm{~d} \\
82.65 \mathrm{~d} \\
75.20 \mathrm{e} \\
\end{array}$ & $\begin{array}{c}99.82 \mathrm{a} \\
100.05 \mathrm{a} \\
86.61 \mathrm{bc} \\
\end{array}$ & $\begin{array}{l}92.01 \mathrm{~b} \\
91.22 \mathrm{~b} \\
87.81 \mathrm{c} \\
\end{array}$ & $\begin{array}{l}87.13 \mathrm{c} \\
83.34 \mathrm{~d} \\
77.82 \mathrm{e} \\
\end{array}$ & $\begin{array}{c}100.52 \mathrm{a} \\
100.64 \mathrm{a} \\
93.27 \mathrm{~b} \\
\end{array}$ \\
\hline & Time of $\mathrm{N}$ application & & & & & \multirow[b]{2}{*}{$\begin{array}{l}82.37 \mathrm{e} \\
82.87 \mathrm{e} \\
83.77 \mathrm{e} \\
82.05 \mathrm{e}\end{array}$} & \\
\hline & $\begin{array}{c}1 / 3(B+P I+B T) \\
1 / 3(T+M T+B T) \\
1 / 2 T+1 / 4 P I+1 / 4 B T \\
1 / 4(T+M T+P I+B T)\end{array}$ & $\begin{array}{l}84.36 \mathrm{de} \\
86.60 \mathrm{~cd} \\
87.86 \mathrm{c} \\
86.25 \mathrm{~cd}\end{array}$ & $\begin{array}{l}78.46 \mathrm{~g} \\
79.24 \mathrm{~g} \\
82.91 \mathrm{ef} \\
80.48 \mathrm{fg}\end{array}$ & $\begin{array}{l}94.01 \mathrm{~b} \\
93.50 \mathrm{~b} \\
95.65 \mathrm{~b} \\
98.81 \mathrm{a}\end{array}$ & $\begin{array}{l}89.78 \mathrm{~cd} \\
90.52 \mathrm{~cd} \\
91.98 \mathrm{c} \\
88.98 \mathrm{~d} \\
\end{array}$ & & $\begin{array}{c}96.77 \mathrm{~b} \\
96.97 \mathrm{~b} \\
97.89 \mathrm{~b} \\
100.94 \mathrm{a}\end{array}$ \\
\hline & \multirow{2}{*}{ Time of $\mathrm{N}$ application } & \multicolumn{6}{|c|}{ N source (2014) } \\
\hline & & \multirow{2}{*}{\multicolumn{2}{|c|}{$\begin{array}{c}\text { A. sulfate } \\
91.15 \mathrm{bc} \\
94.74 \mathrm{a} \\
93.73 \mathrm{a} \\
93.27 \mathrm{ab}\end{array}$}} & \multirow{2}{*}{\multicolumn{2}{|c|}{$\begin{array}{c}\text { Urea } \\
90.31 \mathrm{c} \\
90.33 \mathrm{c} \\
92.50 \mathrm{ab} \\
92.56 \mathrm{ab}\end{array}$}} & \multicolumn{2}{|c|}{ Calcium nitrate } \\
\hline & $\begin{array}{c}1 / 3(\mathrm{~B}+\mathrm{PI}+\mathrm{BT}) \\
1 / 3(\mathrm{~T}+\mathrm{MT}+\mathrm{BT}) \\
1 / 2 \mathrm{~T}+1 / 4 \mathrm{PI}+1 / 4 \mathrm{BT} \\
1 / 4(\mathrm{~T}+\mathrm{MT}+\mathrm{PI}+\mathrm{BT})\end{array}$ & & & & & $\begin{array}{l}87 \\
8 \\
8 \\
8\end{array}$ & \\
\hline & & & & & urce & & \\
\hline & Time of $\mathrm{N}$ anplication & & 2013 & & & 2014 & \\
\hline $\begin{array}{l}\bar{c} \\
\underline{0} \\
\underline{\omega}\end{array}$ & 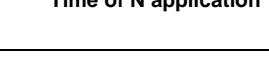 & A. sulfate & Urea & $\begin{array}{c}\text { Calcium } \\
\text { nitrate }\end{array}$ & A. sulfate & Urea & $\begin{array}{c}\begin{array}{c}\text { Calcium } \\
\text { nitrate }\end{array} \\
\end{array}$ \\
\hline 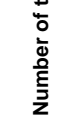 & $\begin{array}{c}1 / 3(B+P I+B T) \\
1 / 3(T+M T+B T) \\
1 / 2 T+1 / 4 P I+1 / 4 B T \\
1 / 4(T+M T+P I+B T)\end{array}$ & $\begin{array}{l}17.52 \mathrm{de} \\
20.15 \mathrm{a} \\
19.97 \mathrm{ab} \\
19.81 \mathrm{ab}\end{array}$ & $\begin{array}{c}17.66 \mathrm{~d} \\
19.18 \mathrm{bc} \\
20.20 \mathrm{a} \\
19.37 \mathrm{ab}\end{array}$ & $\begin{array}{c}18.52 \mathrm{c} \\
17.00 \mathrm{de} \\
17.43 \mathrm{de} \\
16.72 \mathrm{e}\end{array}$ & $\begin{array}{l}22.04 \mathrm{cde} \\
23.52 \mathrm{a} \\
23.23 \mathrm{ab} \\
22.54 \mathrm{abc}\end{array}$ & $\begin{array}{l}21.36 \text { ef } \\
22.96 \mathrm{abc} \\
22.31 \mathrm{bcd} \\
21.95 \mathrm{cde}\end{array}$ & $\begin{array}{l}22.30 \mathrm{bcd} \\
21.49 \mathrm{def} \\
21.60 \mathrm{def} \\
20.93 \mathrm{f}\end{array}$ \\
\hline
\end{tabular}

In a column, means followed by a common letters are not significantly different at $5 \%$ level according to DMRT. $\mathrm{T}$ = beginning of tillering stage, $\mathrm{MT}=$ mid tillering stage, $\mathrm{PI}=$ panicle initiation stage and BT = booting stage

Data in Tables (5) indicated that the interaction between cultivars and nitrogen sources, nitrogen sources and time of nitrogen 
application had significant effect on panicle weight only in 2014 season. Also, the interaction between cultivars and time of nitrogen application, had significant effect on panicle weight only in 2013 season. Egyptian hybrid one and ammonium sulfate gave the highest values of panicle weight. Egyptian hybrid one under nitrogen split $1 / 4 \mathrm{~T}$ $+1 / 4 \mathrm{MT}+1 / 4 \mathrm{PI}+1 / 4 \mathrm{BT}$ gave the heaviest panicle. The highest values panicle weight were obtained with ammonium sulfate added $1 / 4 \mathrm{~T}+1 / 4$ $\mathrm{MT}+1 / 4 \mathrm{PI}+1 / 4 \mathrm{BT}$.

Data in Tables (5) indicated that the interaction between cultivars and nitrogen sources Also, the interaction between nitrogen sources and time of nitrogen application had significant effect on number of filled grains / panicle only in 2014 season. The combination of Egyptian hybrid 1and ammonium sulfate gave the maximum number of filled grains / panicle. The highest values of number of filled grains / panicle were obtained with ammonium sulfate added $1 / 4 \mathrm{~T}+1 / 4 \mathrm{MT}+1 / 4$ $\mathrm{PI}+1 / 4 \mathrm{BT}$.

Data in Tables (5) referred that the interaction between rice cultivars and nitrogen sources, nitrogen sources and time of nitrogen application had significant effect on number of unfilled grains / panicle in both seasons. Also, the interaction between rice cultivars and time of nitrogen application had significant effect on number of unfilled grains / panicle only in 2013 season. The combination of Egyptian hybrid one and calcium nitrate gave the highest values of number of unfilled grains. The combination of Egyptian hybrid one gave the highest values of number of unfilled grains under three equal nitrogen splits $1 / 3 \mathrm{~B}+1 / 3 \mathrm{PI}+1 / 3 \mathrm{BT}$. The combination of calcium nitrate under four equal nitrogen splits $1 / 4 \mathrm{~T}+1 / 4 \mathrm{MT}+1 / 4 \mathrm{PI}+1 / 4$ BT gave the highest values of number of unfilled grains.

Data in Table (6) indicated that the interaction between cultivars and nitrogen sources, cultivars and time of nitrogen application also, nitrogen sources and time of nitrogen application had significant effect on thousand grain weight only in 2013 season. Egyptian hybrid one and ammonium sulfate gave the highest values of thousand grain weight. Egyptian hybrid one under nitrogen split $1 / 4 T+$ $1 / 4 \mathrm{MT}+1 / 4 \mathrm{PI}+1 / 4 \mathrm{BT}$ gave the heaviest thousand grain weight. The highest values thousand grain weight were obtained with ammonium sulfate added $1 / 4 \mathrm{~T}+1 / 4 \mathrm{MT}+1 / 4 \mathrm{PI}+1 / 4 \mathrm{BT}$. 
Table (5): Number of panicles, panicle weight, number of filled grains and number of unfilled grains as affected by the interaction between the studied factors

\begin{tabular}{|c|c|c|c|c|c|c|c|}
\hline \multirow{7}{*}{ 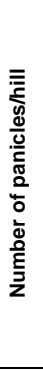 } & \multirow{2}{*}{ N source } & \multicolumn{6}{|c|}{ Rice cultivar (2013) } \\
\hline & & \multicolumn{2}{|c|}{ Giza 178} & \multicolumn{2}{|c|}{ Giza 179} & \multicolumn{2}{|c|}{ EHR1 } \\
\hline & $\begin{array}{c}\text { Ammonium sulfate } \\
\text { Urea } \\
\text { Calcium nitrate }\end{array}$ & \multicolumn{2}{|c|}{$\begin{array}{l}18.92 \mathrm{c} \\
17.90 \mathrm{~d} \\
16.36 \mathrm{e}\end{array}$} & \multicolumn{2}{|c|}{$\begin{array}{l}17.72 \mathrm{~d} \\
16.90 \mathrm{e} \\
14.55 \mathrm{f}\end{array}$} & \multicolumn{2}{|c|}{$\begin{array}{l}21.30 \mathrm{a} \\
20.15 \mathrm{~b} \\
15.06 \mathrm{f}\end{array}$} \\
\hline & \multirow{3}{*}{ Time of $\mathrm{N}$ application } & \multicolumn{6}{|c|}{ N source } \\
\hline & & \multicolumn{3}{|c|}{2013} & \multicolumn{3}{|c|}{2014} \\
\hline & & A. sulfate & Urea & $\begin{array}{l}\text { Calcium } \\
\text { nitrate }\end{array}$ & A. sulfate & Urea & $\begin{array}{l}\text { Calcium } \\
\text { nitrate }\end{array}$ \\
\hline & $\begin{array}{c}1 / 3(\mathrm{~B}+\mathrm{PI}+\mathrm{BT}) \\
1 / 3(\mathrm{~T}+\mathrm{MT}+\mathrm{BT}) \\
1 / 2 \mathrm{~T}+1 / 4 \mathrm{PI}+1 / 4 \mathrm{BT} \\
1 / 4(\mathrm{~T}+\mathrm{MT}+\mathrm{PI}+\mathrm{BT}) \\
\end{array}$ & $\begin{array}{c}16.80 \mathrm{~b} \\
18.00 \mathrm{a} \\
17.34 \mathrm{ab} \\
16.95 \mathrm{~b} \\
\end{array}$ & $\begin{array}{c}15.73 \mathrm{c} \\
16.80 \mathrm{~b} \\
17.23 \mathrm{ab} \\
15.90 \mathrm{c} \\
\end{array}$ & $\begin{array}{l}16.50 \mathrm{bc} \\
13.95 \mathrm{~d} \\
13.43 \mathrm{~d} \\
13.10 \mathrm{~d} \\
\end{array}$ & $\begin{array}{c}18.55 \mathrm{bc} \\
19.80 \mathrm{a} \\
19.82 \mathrm{a} \\
19.08 \mathrm{ab} \\
\end{array}$ & $\begin{array}{r}17.63 \mathrm{~d} \\
18.64 \mathrm{~b} \\
19.21 \mathrm{ab} \\
17.90 \mathrm{~cd} \\
\end{array}$ & $\begin{array}{l}17.65 \mathrm{~d} \\
15.39 \mathrm{e} \\
14.15 \mathrm{f} \\
14.12 \mathrm{f} \\
\end{array}$ \\
\hline \multirow{6}{*}{ 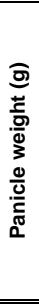 } & \multirow{2}{*}{ N source } & \multicolumn{6}{|c|}{ Rice cultivar (2014) } \\
\hline & & \multirow{2}{*}{\multicolumn{2}{|c|}{$\begin{array}{c}\text { Giza } 178 \\
3.30 \mathrm{~cd} \\
3.17 \mathrm{~d} \\
2.76 \mathrm{e}\end{array}$}} & \multicolumn{2}{|c|}{ Giza 179} & \multicolumn{2}{|c|}{ EHR1 } \\
\hline & $\begin{array}{c}\text { Ammonium sulfate } \\
\text { Urea } \\
\text { Calcium nitrate }\end{array}$ & & & & & & \\
\hline & \multirow[b]{2}{*}{ Time of $\mathrm{N}$ application } & \multicolumn{3}{|c|}{ Rice cultivar (2013) } & \multicolumn{3}{|c|}{$\mathrm{N}$ source (2013) } \\
\hline & & Giza 178 & Giza 179 & EHR1 & A. sulfate & Urea & $\begin{array}{c}\text { Calcium } \\
\text { nitrate }\end{array}$ \\
\hline & $\begin{array}{c}1 / 3(\mathrm{~B}+\mathrm{PI}+\mathrm{BT}) \\
1 / 3(\mathrm{~T}+\mathrm{MT}+\mathrm{BT}) \\
1 / 2 \mathrm{~T}+1 / 4 \mathrm{PI}+1 / 4 \mathrm{BT} \\
1 / 4(\mathrm{~T}+\mathrm{MT}+\mathrm{PI}+\mathrm{BT}) \\
\end{array}$ & $\begin{array}{l}2.77 \mathrm{e} \\
3.21 \mathrm{~cd} \\
3.13 \mathrm{~d} \\
3.18 \mathrm{~cd} \\
\end{array}$ & $\begin{array}{l}2.76 \mathrm{e} \\
3.12 \mathrm{~d} \\
3.30 \mathrm{~cd} \\
3.28 \mathrm{~cd} \\
\end{array}$ & $\begin{array}{c}3.42 \mathrm{c} \\
3.92 \mathrm{~b} \\
4.10 \mathrm{ab} \\
4.33 \mathrm{a} \\
\end{array}$ & $\begin{array}{c}3.12 \mathrm{~d} \\
3.92 \mathrm{~b} \\
4.10 \mathrm{ab} \\
4.33 \mathrm{a} \\
\end{array}$ & $\begin{array}{c}3.13 \mathrm{~d} \\
3.18 \mathrm{~cd} \\
3.30 \mathrm{~cd} \\
3.42 \mathrm{c} \\
\end{array}$ & $\begin{array}{l}3.28 \mathrm{~cd} \\
3.21 \mathrm{~cd} \\
2.77 \mathrm{e} \\
2.76 \mathrm{e} \\
\end{array}$ \\
\hline \multirow{6}{*}{ 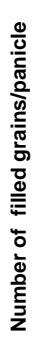 } & \multirow{2}{*}{ N source } & \multicolumn{6}{|c|}{ Rice cultivar (2014) } \\
\hline & & \multirow{2}{*}{\multicolumn{2}{|c|}{$\begin{array}{c}\text { Giza 178 } \\
128.2 \mathrm{~b} \\
124.3 \mathrm{c} \\
119.1 \mathrm{~d} \\
\end{array}$}} & \multirow{2}{*}{\multicolumn{2}{|c|}{$\begin{array}{c}\text { Giza } 179 \\
109.5 \mathrm{e} \\
109.1 \mathrm{e} \\
93.7 \mathrm{f} \\
\end{array}$}} & \multicolumn{2}{|c|}{ EHR1 } \\
\hline & $\begin{array}{c}\text { Ammonium sulfate } \\
\text { Urea } \\
\text { Calcium nitrate }\end{array}$ & & & & & & \\
\hline & \multirow{2}{*}{ Time of $\mathrm{N}$ application } & & & N sou & (2014) & & \\
\hline & & A. 5 & & & & Calc & trate \\
\hline & $\begin{array}{c}1 / 3(B+P I+B T) \\
1 / 3(T+M T+B T) \\
1 / 2 T+1 / 4 P I+1 / 4 B T \\
1 / 4(T+M T+P I+B T)\end{array}$ & & & & & & \\
\hline & & & & Rice & tivar & & \\
\hline & $\mathrm{N}$ source & & 2013 & & & 2014 & \\
\hline & & Giza 178 & Giza 179 & EHR1 & Giza 178 & Giza 179 & EHR1 \\
\hline$\frac{0}{0}$ & $\begin{array}{c}\text { Ammonium sulfate } \\
\text { Urea } \\
\text { Calcium nitrate }\end{array}$ & $\begin{array}{l}15.64 \mathrm{~cd} \\
17.30 \mathrm{c} \\
21.87 \mathrm{~b}\end{array}$ & $\begin{array}{l}14.88 \mathrm{~d} \\
16.06 \mathrm{~cd} \\
22.78 \mathrm{~b}\end{array}$ & $\begin{array}{l}20.82 \mathrm{~b} \\
20.46 \mathrm{~b} \\
38.08 \mathrm{a}\end{array}$ & $\begin{array}{l}14.03 \mathrm{~d} \\
15.50 \mathrm{~cd} \\
21.00 \mathrm{~b}\end{array}$ & $\begin{array}{l}14.18 d \\
14.59 d \\
20.43 b\end{array}$ & $\begin{array}{l}16.68 \mathrm{c} \\
14.81 \mathrm{~d} \\
26.37 \mathrm{a}\end{array}$ \\
\hline$\stackrel{\pi}{2}$ & Time of $\mathrm{N}$ anplication & & & Rice cu & $r(2013)$ & & \\
\hline$\stackrel{\infty}{.}$ & IIme or iv appication & & & & & & \\
\hline 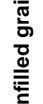 & $\begin{array}{c}1 / 3(\mathrm{~B}+\mathrm{PI}+\mathrm{BT}) \\
1 / 3(\mathrm{~T}+\mathrm{MT}+\mathrm{BT}) \\
1 / 2 \mathrm{~T}+1 / 4 \mathrm{PI}+1 / 4 \mathrm{BT} \\
1 / 4(\mathrm{~T}+\mathrm{MT}+\mathrm{PI}+\mathrm{BT})\end{array}$ & & & & & & \\
\hline$\Xi$ & & & & & $\mathrm{ce}$ & & \\
\hline$\overleftarrow{0}$ & Time of $\mathrm{N}$ application & & 2013 & & & 2014 & \\
\hline 迆 & & A. sulfate & Urea & $\begin{array}{l}\text { Calcium } \\
\text { nitrate }\end{array}$ & A. sulfate & Urea & $\begin{array}{c}\text { Calcium } \\
\text { nitrate }\end{array}$ \\
\hline $\bar{z}$ & $\begin{array}{c}1 / 3(B+P I+B T) \\
1 / 3(T+M T+B T) \\
1 / 2 T+1 / 4 P I+1 / 4 B T \\
1 / 4(T+M T+P I+B T)\end{array}$ & $\begin{array}{l}23.25 \mathrm{c} \\
16.15 \mathrm{ef} \\
16.01 \mathrm{ef} \\
13.04 \mathrm{~g}\end{array}$ & $\begin{array}{l}24.05 \mathrm{c} \\
17.13 \mathrm{e} \\
16.63 \mathrm{e} \\
13.95 \mathrm{fg}\end{array}$ & $\begin{array}{l}35.01 \mathrm{a} \\
29.15 \mathrm{~b} \\
25.41 \mathrm{c} \\
20.75 \mathrm{~d}\end{array}$ & $\begin{array}{l}19.58 \mathrm{c} \\
14.63 \mathrm{~d} \\
14.33 \mathrm{~d} \\
11.33 \mathrm{e}\end{array}$ & $\begin{array}{l}19.62 \mathrm{c} \\
14.33 \mathrm{~d} \\
14.08 \mathrm{~d} \\
11.83 \mathrm{e}\end{array}$ & $\begin{array}{l}30.83 \mathrm{a} \\
24.08 \mathrm{~b} \\
19.58 \mathrm{c} \\
15.91 \mathrm{~d}\end{array}$ \\
\hline
\end{tabular}

In a column, means followed by a common letters are not significantly different at $5 \%$ level according to DMRT. $\mathrm{T}$ = beginning of tillering stage, $\mathrm{MT}=$ mid tillering stage, $\mathrm{PI}=$ panicle initiation stage and BT = booting stage 
Data in Table (6) indicated that the interaction between nitrogen sources and times of nitrogen application had significant effect on biological yield (ton / ha ${ }^{-1}$ ) in both seasons. The highest values biological yield were obtained with ammonium sulfate added $1 / 4 \mathrm{~T}+1 / 4 \mathrm{MT}+1 / 4 \mathrm{PI}+1 / 4 \mathrm{BT}$.

Data in Table (6) referred that the interaction between rice cultivars and nitrogen sources had significant effect on grain yield (ton $/ \mathrm{ha}^{-1}$ ) in both seasons study. Also, the interaction between rice cultivars and times of nitrogen application, nitrogen sources and times of nitrogen application had significant only in 2014 season. The combination Egyptian hybrid 1and ammonium sulfate gave the highest values of grain yield. The combination of Egyptian hybrid one under nitrogen splits in four dosed as $\mathrm{T}+\mathrm{MT}+\mathrm{PI}+\mathrm{BT}$ gave the highest values of grain yield. The combination of ammonium sulfate under nitrogen splits in four dosed as $\mathrm{T}+\mathrm{MT}+\mathrm{PI}+\mathrm{BT}$ gave the highest values of grain yield.

Table (6): 1000-grain weight, biological and grain yields as affected by the interaction between the studied factors

\begin{tabular}{|c|c|c|c|c|c|c|c|}
\hline \multirow{6}{*}{ 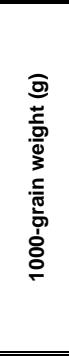 } & \multirow{2}{*}{ N source } & \multicolumn{6}{|c|}{ Rice cultivar (2013) } \\
\hline & & \multicolumn{2}{|c|}{ Giza 178} & \multicolumn{2}{|c|}{ Giza 179} & \multicolumn{2}{|c|}{ EHR1 } \\
\hline & $\begin{array}{c}\text { Ammonium sulfate } \\
\text { Urea } \\
\text { Calcium nitrate }\end{array}$ & \multicolumn{2}{|c|}{$\begin{array}{l}19.33 \mathrm{e} \\
19.21 \mathrm{e} \\
16.97 \mathrm{f}\end{array}$} & \multicolumn{2}{|c|}{$\begin{array}{l}22.48 \mathrm{c} \\
22.72 \mathrm{bc} \\
22.85 \mathrm{bc}\end{array}$} & \multicolumn{2}{|c|}{$\begin{array}{l}24.14 \mathrm{a} \\
23.16 \mathrm{~b} \\
21.83 \mathrm{~d}\end{array}$} \\
\hline & \multirow[b]{2}{*}{ Time of $\mathrm{N}$ application } & \multicolumn{3}{|c|}{ Rice cultivar (2013) } & \multicolumn{3}{|c|}{ N source $(2013)$} \\
\hline & & Giza 178 & Giza 179 & EHR1 & A. sulfate & Urea & $\begin{array}{c}\text { Calcium } \\
\text { nitrate }\end{array}$ \\
\hline & $\begin{array}{c}1 / 3(\mathrm{~B}+\mathrm{PI}+\mathrm{BT}) \\
1 / 3(\mathrm{~T}+\mathrm{MT}+\mathrm{BT}) \\
1 / 2 \mathrm{~T}+1 / 4 \mathrm{PI}+1 / 4 \mathrm{BT} \\
1 / 4(\mathrm{~T}+\mathrm{MT}+\mathrm{PI}+\mathrm{BT}) \\
\end{array}$ & $\begin{array}{l}17.95 \mathrm{f} \\
18.17 \mathrm{ef} \\
18.56 \mathrm{e} \\
19.33 \mathrm{~d} \\
\end{array}$ & $\begin{array}{c}22.18 \mathrm{c} \\
22.47 \mathrm{bc} \\
23.00 \mathrm{ab} \\
23.10 \mathrm{a} \\
\end{array}$ & $\begin{array}{c}22.50 \mathrm{bc} \\
23.16 \mathrm{a} \\
23.26 \mathrm{a} \\
23.26 \mathrm{a} \\
\end{array}$ & $\begin{array}{l}20.72 \text { def } \\
21.94 \mathrm{bc} \\
22.37 \mathrm{~b} \\
22.91 \mathrm{a} \\
\end{array}$ & $\begin{array}{l}20.98 \mathrm{~d} \\
21.49 \mathrm{c} \\
22.02 \mathrm{~b} \\
22.29 \mathrm{~b} \\
\end{array}$ & $\begin{array}{c}20.93 \text { de } \\
20.38 \mathrm{f} \\
20.43 \text { ef } \\
20.46 \text { de } \\
\end{array}$ \\
\hline \multirow{4}{*}{ 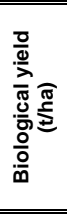 } & \multirow{3}{*}{ Time of $\mathrm{N}$ application } & \multicolumn{6}{|c|}{ N source } \\
\hline & & \multicolumn{3}{|c|}{2013} & \multicolumn{3}{|c|}{2014} \\
\hline & & A. sulfate & Urea & $\begin{array}{l}\text { Calcium } \\
\text { nitrate }\end{array}$ & A. sulfate & Urea & $\begin{array}{c}\text { Calcium } \\
\text { nitrate }\end{array}$ \\
\hline & $\begin{array}{c}1 / 3(\mathrm{~B}+\mathrm{PI}+\mathrm{BT}) \\
1 / 3(\mathrm{~T}+\mathrm{MT}+\mathrm{BT}) \\
1 / 2 \mathrm{~T}+1 / 4 \mathrm{PI}+1 / 4 \mathrm{BT} \\
1 / 4(\mathrm{~T}+\mathrm{MT}+\mathrm{PI}+\mathrm{BT}) \\
\end{array}$ & $\begin{array}{c}12.04 \mathrm{~g} \\
16.38 \mathrm{ab} \\
15.06 \mathrm{~d} \\
16.95 \mathrm{a} \\
\end{array}$ & $\begin{array}{c}12.12 \mathrm{~g} \\
15.31 \mathrm{~cd} \\
15.11 \mathrm{~d} \\
15.97 \mathrm{bc}\end{array}$ & $\begin{array}{l}12.54 \mathrm{~g} \\
13.33 \mathrm{f} \\
13.53 \mathrm{f} \\
14.37 \mathrm{e}\end{array}$ & $\begin{array}{c}13.17 \mathrm{f} \\
17.78 \mathrm{ab} \\
16.51 \mathrm{bcd} \\
18.93 \mathrm{a} \\
\end{array}$ & $\begin{array}{c}13.06 \mathrm{f} \\
16.13 \mathrm{~cd} \\
16.04 \mathrm{~cd} \\
16.81 \mathrm{bc}\end{array}$ & $\begin{array}{c}12.92 \mathrm{f} \\
14.67 \mathrm{e} \\
14.26 \mathrm{ef} \\
15.22 \mathrm{de}\end{array}$ \\
\hline \multirow{7}{*}{ 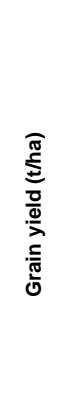 } & \multirow{3}{*}{ N source } & \multicolumn{6}{|c|}{ Rice cultivar } \\
\hline & & \multicolumn{3}{|c|}{2013} & \multicolumn{3}{|c|}{2014} \\
\hline & & Giza 178 & Giza 179 & EHR1 & Giza 178 & Giza 179 & EHR1 \\
\hline & $\begin{array}{c}\text { Ammonium sulfate } \\
\text { Urea } \\
\text { Calcium nitrate } \\
\end{array}$ & $\begin{array}{c}6.72 \mathrm{c} \\
6.67 \mathrm{~cd} \\
5.77 \mathrm{c} \\
\end{array}$ & $\begin{array}{l}6.43 \mathrm{~d} \\
6.71 \mathrm{c} \\
5.31 \mathrm{f} \\
\end{array}$ & $\begin{array}{l}7.98 \mathrm{a} \\
7.61 \mathrm{~b} \\
5.96 \mathrm{e} \\
\end{array}$ & $\begin{array}{l}6.98 \mathrm{c} \\
6.95 \mathrm{c} \\
5.93 \mathrm{e} \\
\end{array}$ & $\begin{array}{l}6.59 \mathrm{~d} \\
6.85 \mathrm{c} \\
5.62 \mathrm{f} \\
\end{array}$ & $\begin{array}{l}8.35 \mathrm{a} \\
7.80 \mathrm{~b} \\
6.37 \mathrm{~d} \\
\end{array}$ \\
\hline & \multirow[b]{2}{*}{ Time of $\mathrm{N}$ application } & \multicolumn{3}{|c|}{ Rice cultivar (2014) } & \multicolumn{3}{|c|}{ N source (2014) } \\
\hline & & Giza 178 & Giza 179 & EHR1 & A. sulfate & Urea & $\begin{array}{l}\text { Calcium } \\
\text { nitrate }\end{array}$ \\
\hline & $\begin{array}{c}1 / 3(\mathrm{~B}+\mathrm{PI}+\mathrm{BT}) \\
1 / 3(\mathrm{~T}+\mathrm{MT}+\mathrm{BT}) \\
1 / 2 \mathrm{~T}+1 / 4 \mathrm{PI}+1 / 4 \mathrm{BT} \\
1 / 4(\mathrm{~T}+\mathrm{MT}+\mathrm{PI}+\mathrm{BT})\end{array}$ & $\begin{array}{l}5.98 \mathrm{~h} \\
7.18 \mathrm{c} \\
665 \mathrm{de} \\
6.67 \mathrm{de}\end{array}$ & $\begin{array}{l}6.13 \mathrm{gh} \\
6.59 \mathrm{ef} \\
6.35 \mathrm{fg} \\
6.36 \mathrm{fg}\end{array}$ & $\begin{array}{c}6.89 \mathrm{~d} \\
7.73 \mathrm{ab} \\
7.49 \mathrm{~b} \\
7.93 \mathrm{a}\end{array}$ & $\begin{array}{c}6.45 \mathrm{de} \\
7.94 \mathrm{a} \\
7.24 \mathrm{c} \\
7.60 \mathrm{~b}\end{array}$ & $\begin{array}{l}6.52 \mathrm{~d} \\
7.36 \mathrm{bc} \\
7.36 \mathrm{bc} \\
7.57 \mathrm{~b}\end{array}$ & $\begin{array}{l}6.02 \mathrm{fg} \\
6.00 \mathrm{~g} \\
5.89 \mathrm{~g} \\
5.79 \mathrm{~g}\end{array}$ \\
\hline
\end{tabular}

In a column, means followed by a common letters are not significantly different at $5 \%$ level according to DMRT. $\mathrm{T}$ = beginning of tillering stage, $\mathrm{MT}=$ mid tillering stage, $\mathrm{PI}=$ panicle initiation stage and BT = booting stage 


\section{REFERENCES}

Assefa M., F. Getnet, A. Menzir and H. Kurabachew (2009). Response of upland rice for nitrogen and phosphorus fertilizers on verticals of Pawe area. In: Proceeding of the $10^{\text {th }}$ Conference of the Ethiopian Soc. Soil Sci. hold during 25-27 March 2009, Addis Ababa, Ethiopia.

Balasubramnain, R. (2002). Response of hybrid rice (Oryza sativa L.) to level and time of nitrogen application. Indian J. Agro., 47(2):203206.

Chien, S. H., Gearhart, M. Mercedes and V.Saven (2011). Comparison of ammonium sulfate with other nitrogen and sulfur fertilizer in increasing crop production and minimizing environmental impact. Soil. Science. J. 76- (7): 327-335.

Duncan, B . D . (1955) Multiple Range and Multiple F. Test. Biometrics. 11: 1-42.

Edwin, L., J. Krisjnarajan and M. Premsekhar (2004). Irrigation and nitrogen application schedules for hybrid ADTRH1 rice (Oryza sativa L.,) in Tamil Nadu. J. Agro., 49 (1):37-39.

El-Refaee, I. S. H. (2007). Effect of cut-off irrigation date on grain yield and quality of some Egyptian rice cultivars. J. Agric. Res. Kafr El-Sheikh Univ., 33(1):123-139.

Fageria, N. K., A. B. Dos Santos and A. M. Coelho (2011). Growth, yield and yield component of lowland rice as influenced by ammonium sulfate and urea fertilization. J.of Plant Nutr, 34(3):371-386.

Fageria, N. K., and V. C. Baligar (2005). Enhancing nitrogen use efficiency in crop plants. Advan in Agro 80:97-185.

Gomez, K. A. and A.A. Gomez (1984). Statistical Procedures for Agricultural Researches. 2nd Ed., John Wiley \& Sons. U.S.A. Pak.

Hembram, S. P; Bandyopadhyay; P. K. Jana; D. Dutta and D.Ray. (2001). Effect of depth and time of irrigation and fertilizer on yield attributes, yield and production economics of hybrid rice (Oryza sativa L.) Crop. Res. 21(1):11-14.

Jan, M.T., M. J. Khan., A. Arif., M. Shafi and M. Farmanullah. (2010). Wheat nitrogen indices response to nitrogen source and application time. Pak. J. Bot. 42(6), 4267-4279.

RRTC (2014). Rice research and training center, Annual report agronomy. Sakha, Kafr El-Sheikh, Egypt.

Tao, S. S., X. C. Xiang and X. F. Zhang. (2002) Studies on application of nitrogen fertilizer in scattered-transplanting cultivation of large panicle hybrid rice. Southwest China J. Agric. Sci. 15(3):39-42.

Yang, J; B.Su; Z. Wang; Y. Lang and Q. Zhu.(1999). Characteristics and physiology of grain filling in inter-subspecfic hybrid rice. Chinese Agric. Sci. J. 40 (3): 1644-1655.

Zayed. B. A., I. S. El-Refaee and S. E. M. Sedeek (2010). Response of different rice varieties to phosphorus fertilizer under newly 
reclaimed saline soil J. Plant. Prod. Mansoura Univ., 1(11)14791493.

Zayed, B. A., S. M. Shehata., A. A. Abd Allah and W. M. El-Khoby (2007). Growth and grain yield of hybrid and traditional rice as affected by splitting nitrogen under saline soil conditions. J. Agric. Res. Kafer El-Sheikh Univ., 33(4)787-806.

Zayed B. A. (2012). Efficiency of different sulfur fertilizer sources in increasing hybrid rice productivity under saline soil conditions. Egypt. J. agric. Res., 90 (4): 275-287.

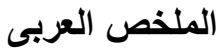

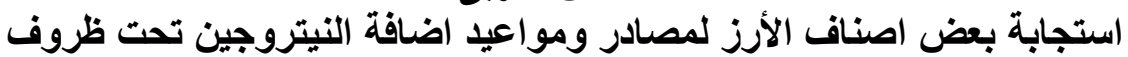 الارض الملحيه}

عبد العزيز جلال عبد الحافظ1، بسيوني عبد الرازق زايد2 ، ابراهيم سعد الاجوي 1 أحمد

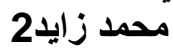

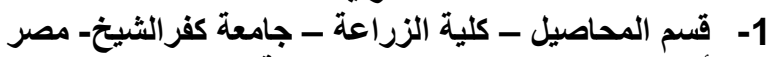

2- قسم بحوث الأرز - معهد بحوث المحاصيل الحقلية ـ مركز البحوث الزئة الزراعية ـ مصر

أجريت تجربتان حقليتان بالمزرعة البحثية بمحطة بحوث السرو الزراعيه بدمياط مصر خلال موسمي

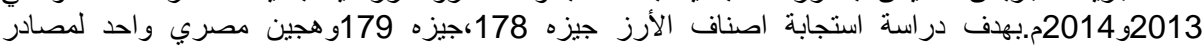

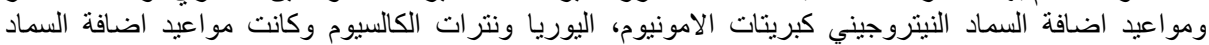

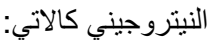

م-1 تجزئة ألسماد في ثلاث دفعات منساويه الثلث على الثر اقي خلطا بالتربه الجافه و عند مرحلة بداية تكوين

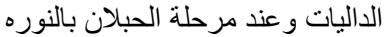

م2- تجزئة السماد في ثلاثل دفعات متساويه عند بداية التفريع وعند مرحلة التفريع المتوسط وعند مرحلة

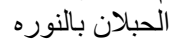

م3- تجزئة السماد في ثلاث دفعات النصف عند بداية التفريع وربع الكميه عند مرحلة بداية تكوين الداليات

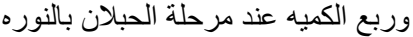
م4- تجزئة السماد في اربع دفعات متساويه عند بداية بلداية التفريع و عند مرحلة التفريع المتوسط و عند مرحلة بداية

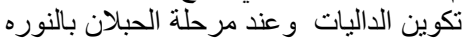

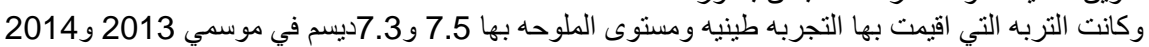

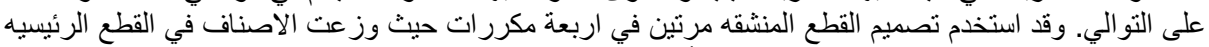

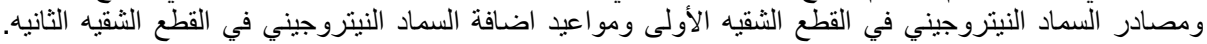

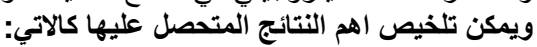

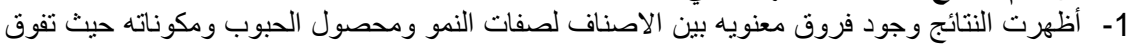

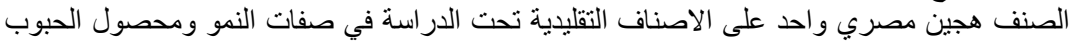

$$
\text { ومكوناته في موسمي ألدر اسها. }
$$

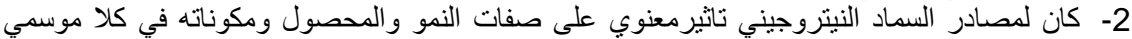

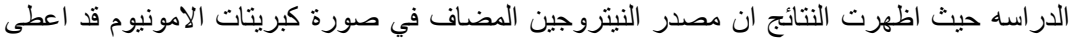
اعلى محصول للحبوب ويليه مصدر السماد النيتروجيني المضاف في صورة اليوريا في كلا موسي الإني

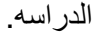

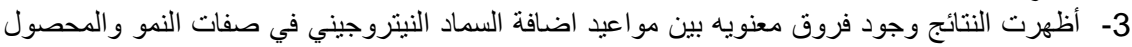

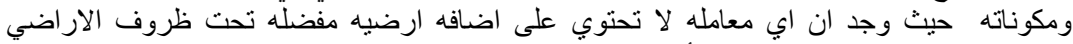

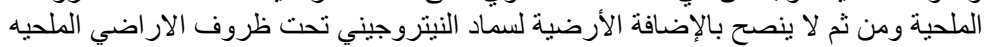

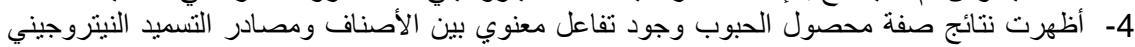

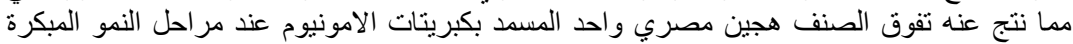

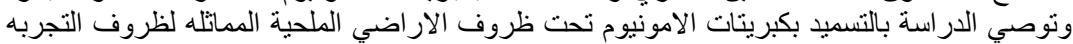
مع تجزئة كمية لسماد على اربعة دفعات عند مر احل النمو الرئيسية حتى بداية طرد الداليات. 